ISPRS Annals of the Photogrammetry, Remote Sensing and Spatial Information Sciences, Volume I-4, 2012 XXII ISPRS Congress, 25 August - 01 September 2012, Melbourne, Australia

\title{
A STUDY OF URBAN INTENSIVE LAND EVALUATING SYSTEM
}

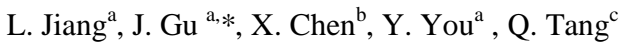

${ }^{a}$ Key Laboratory of Land Resources Evaluation and Monitoring in Southwest, Ministry of Education, Sichuan Normal University, 610068 Chengdu, Sichuan, China - gjr@sicnu.edu.cn, annygu126@126.com, yuyouxiang1984@gmail.com.

${ }^{\mathrm{b}}$ The Bureau of Land Resources Chengdu, 610072 Chengdu, Sichuan, China - shineway@sina.com.

${ }^{\mathrm{c}}$ Information Centre of the Bureau of Land Resources Chengdu, 610072 Chengdu, Sichuan, China tangqiao_2008@yahoo.com.cn.

\section{Commission IV/1: Geospatial Data Infrastructure}

KEY WORDS: Evaluation System, Urban Intensive Land Use, Dynamic Data Extraction Method, WebGIS, B/S.

\begin{abstract}
:
The contradiction of land supply and demand is becoming increasingly prominent in China. The increasing efficiency of land use is an important means to resolve the conflict. We propose a scientific approach for promoting the urban intensive land use. In this paper, an evaluation system of urban intensive land use is programmed. It is designed to change the manual way of collecting index data and building index system to a dynamical way. The system improves the efficiency and accuracy of the evaluation of urban intensive land use. It achieves intensive evaluation on three scales: macro-level, medium-level and micro-level.

We build two data extraction methods. One is XML-based meta-data exchange method that obtains index data from the cadastral database. Another is data monitoring method that writes the index data to the evaluation database at real time. Database technologies are used to calculate index values and build index systems dynamically. GIS technologies are use to achieve three scales evaluation of urban intensive land use.
\end{abstract}

\footnotetext{
* Corresponding author is with the Key Laboratory of Land Resources Evaluation and Monitoring in Southwest (Emailannygu126@126.com)
} 


\section{INTRODUCTION}

Evaluation of urban intensive land use involves geography, economics, planning, etc. Rossiter (1990) made a microcomputer program (ALES) that allowed land evaluators to build their own knowledge-based systems with which they can compute the physical and economic suitability of land map units in accordance with the FAO's Framework for Land Evaluation. They built decision trees to express inferences from land characteristics to land qualities, from land qualities to predicted yields, and from land qualities to overall physical suitability. A representative model is described in the system. Johnson (1991) outlined a new method based on soil potential ratings, which incorporates biophysical crop simulation modelling, expert systems and risk analysis. The technique is able to integrate biophysical and economic data in a measure which can be readily computed, updated and communicated to land managers. Davidson (1994) went on to investigate land evaluation by using a GIS. A land suitability map for receipt of sewage was also derived. A comparison of results from using Boolean and fuzzy set methodologies highlighted the advantages of the latter, although critical decisions are required on choice of membership functions and weights which have a major effect on the results. Machin J.(1995) made an expert land evaluation system (MicroLEIS) along with a geographic information system (IDRISI), which are used to develop a computerized spatial database in order to suggest suitable land management strategies for environment conservation. Other key contributors are Fu (1997), Rosa (2004) and Thapa (2008). In china, the urban intensive land use evaluation study started later. GIS technology was used to develop information systems in the 1990s to improve work efficiency of government and business. Some papers focus on this area, such as Gao (2003), Xu (2006) and Qiao (2007). They developed the systems by using developing components of GIS software and database. The systems' main functions are to manage the land data efficiently, update a database immediately, query and analyze data conveniently, and gather the evaluation result based on well-established model for the spatial analysis. Their systems are based on $\mathrm{C} / \mathrm{S}$ structure. The index systems are designed statically that cannot be changed and the data had to be collected and inputted manually. The utilization of GIS technology is mainly for graphic display, results output and simple query.

With the rapid development of GIS and database technology, our system is based on B/S, GIS and spatial database. It is a distributed system. The index data are collected dynamically. The index system can be adjusted based on demand. The GIS technologies are used to spatial analysis more.

\section{DATABASE DESIGN}

The system database includes index data, graphic data, and data that the system needs to run regularly. There are two types of data that are stored in Oracle 10G: spatial data and attribute data. The data are land parcel-based. This parcel data allow explicit cross-referencing of other spatial features such as planning and political boundaries. Parcel data generate composite representations of the attributes of social-economic data.

A synthetic database that represents only selected characteristics of GDP, population, jobs, real estate, and locations allow for coverage of the entire and permit macro, medium, and micro calculations. The database logical design was as follows:

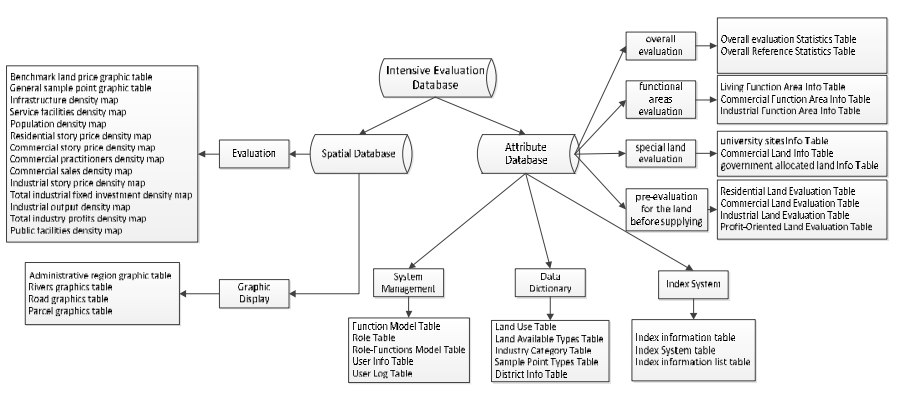

Figure 1. Database Logical Design

\section{SYSTEM DESIGN}

The system implements the complex functions and updates the data as the data source changed. The system concept framework is as follows:

1.Using a data monitoring algorithm monitors data changing in the cadastral database and extract changed data dynamically;

2.Using a spatial discrimination method distributes the socio-economic data to cover the whole research area;

3.Builds the index system based on demands.

The system builds on three-tier $\mathrm{B} / \mathrm{S}$ architectures. The data extraction methods and GIS technologies were accomplished in the business logic layer.

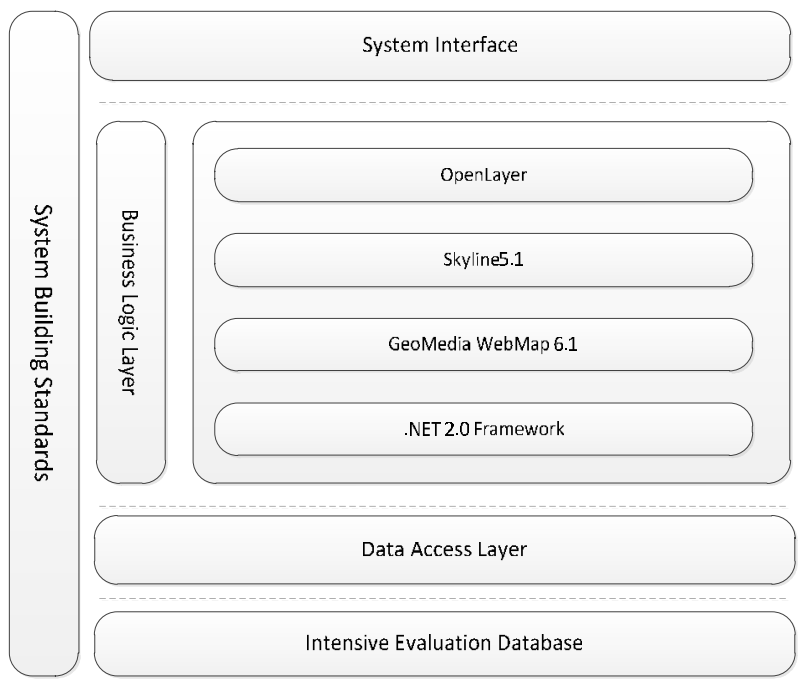

Figure 2. System architecture diagram

\section{SYSTEM IMPLEMENTATION}

The system functions are composed four sub systems. The system management, data management, index management and evaluation management.

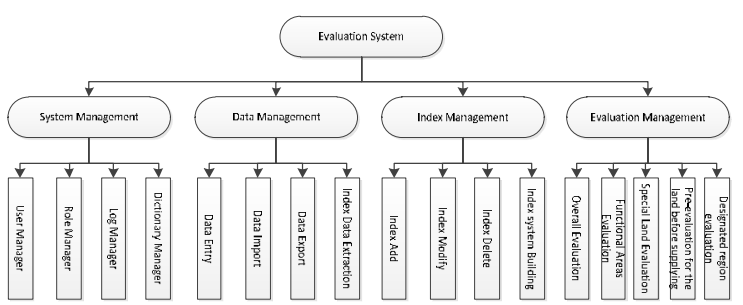

Figure 3. System features model design 
Our system solves three problems in evaluation of urban intensive land use.

First, Index data extraction is built to extract the evaluation data from the cadastral database. Cadastral database is an Oracle database maintained by Land and Resource Information Centre in Chengdu. The database is protected that nobody can do any writing operations in it. Therefore, we used metadata and XML technologies to build a flexible data extracting method. Metadata is used to map data tables in different databases. XML is used to pull data from cadastral database. ADO technology is used to read and write the data. The external database tools are highly scalable (Figure 4). Both data and the metadata mapping information are recorded in the XML files. When new tables and fields are added to the source database, the XML files modify to extract the new data or the changed data. The data monitoring function captures changed data from the source database and updates the records in the target database automatically.

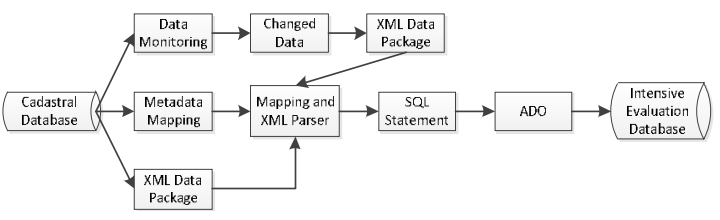

Figure 4. Metadata, XML data monitoring extraction method

Second, index system builds dynamically. The index system contained two types of indexes: national regulation indexes and customized indexes. At intensive evaluation, we build an index system that contains all or some national regulation indexes and some customized. Because different experts have the different understanding of important index of intensive evaluation, experts can have the specify index system by themselves, not just using one index system. In this function, they can add, delete, and modify the indexes, yardstick of index values and weights. The index system can be build easily and intelligently.

1. Three tables are designed in the database: index information table, index system table and index information list table (Figure 5).

2. Index information table saves the entire index information including national regular indexes and customized indicators.

3. Index System table stores the current and historical data.

4. Index information list table contains all the indexes value, reasonable value, and weights.

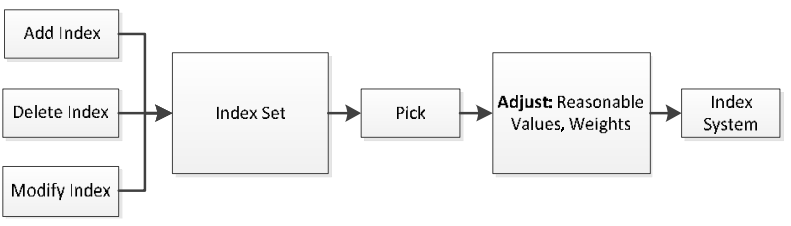

Figure 5. Dynamically build index system model diagram

Several Oracle procedures are created to calculate the index values. These procedures calculate the index value with the basic data attribute table, then save the index value in the index information list table (Figure 6).

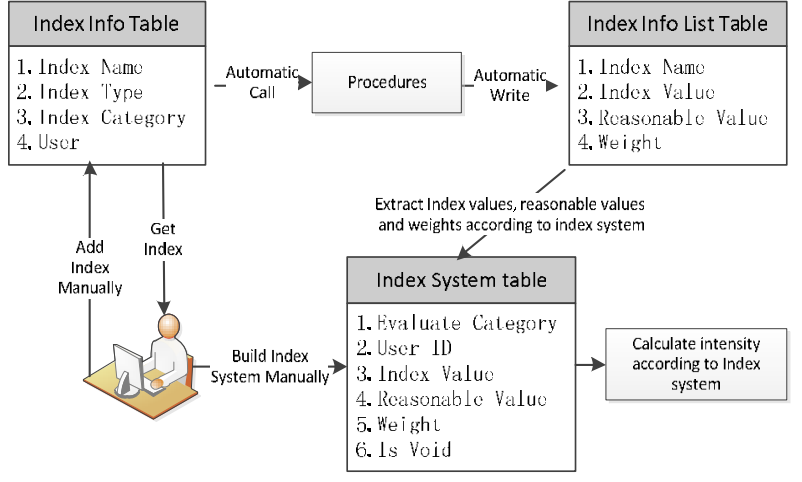

Figure 6. Dynamically build index system diagram

Third, the system calculates five intensive evaluation values: overall evaluation values, functional areas evaluation values, special land use evaluation values, unsupplied land preevaluation values, and selected areas evaluation values (Figure 7).

1. Overall evaluation values calculate the city intensive value. The results display in charts.

2. Functional areas evaluation values calculate the intensive values of the residential, commercial and industrial functional areas. The results display in charts and functional areas $3 \mathrm{D}$ views.

3. Special land use evaluation values calculate the intensive values of the residential-commercial, government and university functional areas. The results display in charts.

4. Unsupplied land pre-evaluation values calculate the intensive values of the residential, commercial, industrial and residential-commercial lots before they will be supplied. The results show in charts.

5. Selected area evaluation values calculate the intensive value of the residential, commercial and industrial areas that selected by users. The results show in charts.

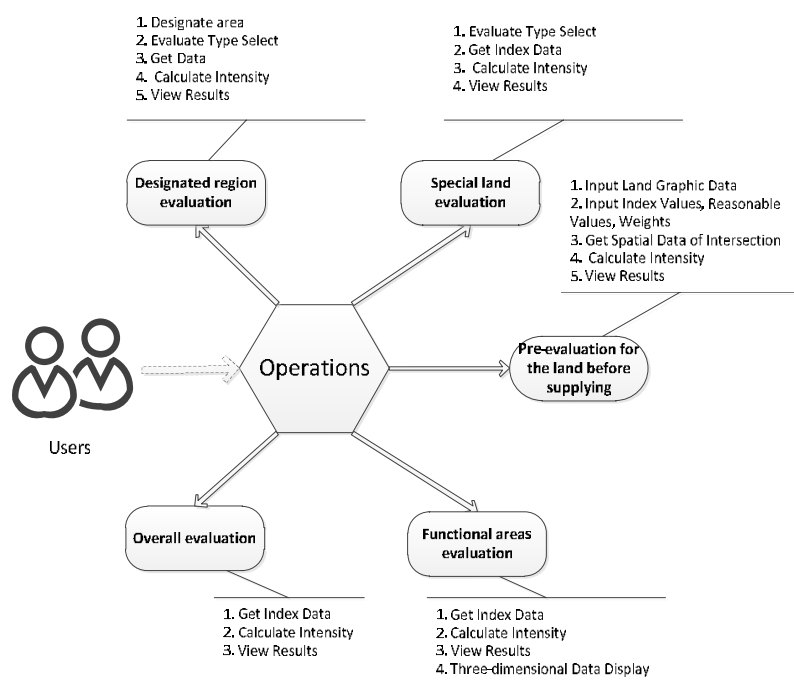

Figure 7. Intensive evaluate function diagram

\section{CONCLUSIONS}

We developed an urban intensive land evaluation system. The system programs using $\mathrm{C} \#$ and bases on metadata, database and GIS technologies. The system collects the index data automatically, builds evaluation index system the dynamically, 
and displays in three-dimensional. It provides more scientific approaches for promoting the intensive land use that calculates the intensive value whenever necessary, so the governments monitor the intensive land use changing at all time. The research accomplishes the following works:

1. Manage the data centralized and maintain data online by using Web GIS technology to reduce the users' complexity of operating professional GIS system.

2. Establish a mechanism to dynamically update the index data. The metadata and XML methods get large quantity of index data in a short time and update the data to the intensive evaluation database without delay when the source database were changed.

3. Build the index system dynamically for different purposes. It increases flexibility in index systems establishing. Users can build the specified index system to meet the different demands.

\section{REFERENCES}

1. Davidson, D. A., Theocharopoulos, S. P., and Bloksma, R. J., 1994. A land evaluation project in Greece using GIS and based on Boolean and fuzzy set methodologies. International Journal of Geographical Information systems, 8(4), pp. 369-384

2. Fu, B., Chen, L., Ma, C., 1997. The Index System and Method of Land Sustainable Use Evaluation. Journal of natural resources, 12(2), pp. 112-118.

3. Gao, P., Gao, Q., 2003. Development and Utilization of City Intensive Land Use Potential Evaluation System. Northeast Surveying and Mapping, 26(2), pp.49-51.(In Chinese)

4. Johnson, A. K. L., Cramb, R. A.,1991. Development of a simulation based land evaluation system using crop modelling, expert systems and risk analysis. Soil Use and Man- agement, 7(4), pp. 239-246.

5. Qiao, W., Chen, J., 2007. The Establishment and Study on Intensive Urban Land Utilization and Potentialities Evaluation Information System. Journal of Nanjing Xiaozhuang College, 6, pp.82-86.(In Chinese)

6. Machin, J., Navas,A.,1995. Land evaluation and conservation of semiarid agrosystems in Zaragoza (ne Spain) using an expert evaluation system and GIS. Land Degradation \& Development, 6(4), pp. 203-214

7. Rosa, D.,_Mayol, F., Diaz-Pereira, E., ect., 2004.A land evaluation decision support system (MicroLEIS DSS) for agricultural soil protection With special reference to the Mediterranean region. Environmental Modelling \& Software, 19, pp. $929-942$.

8. Rossiter, D.G., 1990. ALES: a framework for land evaluation using a microcomputer. Soil Use and Management, 6(1), 7-20.

9. Thapa, R.B., Murayama, Y., 2008. Land evaluation for peri-urban agriculture using analytical hierarchical process and geographic information system techniques: A case study of Hanoi. Land Use Policy, 25(2), 225 - 239.

10. Xu, X., Zhang, J., 2006. Analysis of Information System Construction for the Evaluation of Urban Land Intensive Use and Potential. Journal of Liaocheng University, 19(3), pp.72.(In Chinese)

11. Zhang, Q., Sun, Z., 2003. Design of Evaluating Land Use Intensity Potential in Urban Area Information System. Journal of Test and Measurement and control Technology, 17(4), pp.346-349. (In Chinese) 